\title{
Generalized Contraction and Invariant Approximation Results on Nonconvex Subsets of Normed Spaces
}

\author{
Mujahid Abbas, ${ }^{1}$ Basit Ali, ${ }^{2}$ and Salvador Romaguera ${ }^{3}$ \\ ${ }^{1}$ Department of Mathematics and Applied Mathematics, University of Pretoria, Lynnwood Road, Pretoria 0002, South Africa \\ ${ }^{2}$ Department of Mathematics, Syed Babar Ali School of Science and Engineering, Lahore University of Management Sciences, \\ Lahore 54792, Pakistan \\ ${ }^{3}$ Instituto Universitario de Matemática Pura y Aplicada, Universitat Politècnica de València, Camí de Vera s/n, 46022 Valencia, Spain \\ Correspondence should be addressed to Salvador Romaguera; sromague@mat.upv.es
}

Received 6 December 2013; Accepted 18 January 2014; Published 26 February 2014

Academic Editor: Wei-Shih Du

Copyright (C) 2014 Mujahid Abbas et al. This is an open access article distributed under the Creative Commons Attribution License, which permits unrestricted use, distribution, and reproduction in any medium, provided the original work is properly cited.

Wardowski (2012) introduced a new type of contractive mapping and proved a fixed point result in complete metric spaces as a generalization of Banach contraction principle. In this paper, we introduce a notion of generalized F-contraction mappings which is used to prove a fixed point result for generalized nonexpansive mappings on star-shaped subsets of normed linear spaces. Some theorems on invariant approximations in normed linear spaces are also deduced. Our results extend, unify, and generalize comparable results in the literature.

\section{Introduction and Preliminaries}

One of the most basic and important results in metric fixed point theory is the Banach contraction principle due to Banach [1]. It states that if $(X, d)$ is a complete metric space and $f: X \rightarrow X$ satisfies

$$
d(f x, f y) \leq k d(x, y)
$$

for all $x, y \in X$, with $k \in(0,1)$, then $f$ has a unique fixed point. This theorem that has been extended in many directions (see, e.g., [2-6]) has many applications in mathematics and other related disciplines as well (see, e.g., [7-9]).

Meinardus [10] and Brosowski [11] employed fixed point theory to obtain invariant approximation results in normed linear spaces. A number of authors generalized their results (see $[12-18]$ and the references therein). On the other hand, Dotson [19] extended Banach's contraction principle for nonexpansive mappings on star-shaped subsets of Banach spaces and proved Brosowski-Meinardus type theorems on invariant approximations. L. A. Khan and A. R. Khan [20] generalized Dotson's results on star shaped subsets of $\mathrm{p}$ normed spaces.
Recently, Wardowski [21] introduced a new contractive mapping called an $F$-contraction and proved some fixed point theorems in complete metric spaces. In this paper we introduce a notion of generalized $F$-contraction mappings which is used to prove a fixed point result for generalized nonexpansive mappings on star shaped subsets of normed linear spaces. Some theorems on invariant approximations in normed linear spaces are deduced. Our results extend, unify, and generalize comparable results in $[10,11,19,20]$. Some illustrative examples are also presented.

Next we give some definitions which will be used in the sequel. The letters $\mathbb{R}_{+}, \mathbb{R}$ will denote the set of positive real numbers and the set of real numbers, respectively.

Let $F$ be the collection of all mappings $F: \mathbb{R}_{+} \rightarrow \mathbb{R}$ which satisfy the following conditions:

(C1) $F$ is strictly increasing, that is, for all $\alpha, \beta \in \mathbb{R}_{+}$such that $\alpha<\beta \Rightarrow F(\alpha)<F(\beta)$;

(C2) for every sequence $\left\{\alpha_{n}\right\}_{n \geq 1}$ of positive numbers $\lim _{n \rightarrow \infty} \alpha_{n}=0$ if and only if $\lim _{n \rightarrow \infty} F\left(\alpha_{n}\right)=-\infty$;

(C3) there exists a $k \in(0,1)$ such that $\lim _{\alpha \rightarrow 0^{+}} \alpha^{k} F(\alpha)=0$. 
Definition 1 (see [21]). Let $(X, d)$ be a metric space and $F \in F$. A mapping $f: X \rightarrow X$ is said to be an $F$-contraction on $X$ if there exists a $\tau>0$ such that

$$
d(f x, f y)>0 \Longrightarrow \tau+F(d(f x, f y)) \leq F(d(x, y))
$$

for all $x, y \in X$.

Remark 2 (see [21]). Every F-contraction mapping is continuous.

Motivated by the work of Wardowski [21] and by Theorem 4 of [22], we give the following definition.

Definition 3. Let $(X, d)$ be a metric space and $F \in F$. A mapping $f: X \rightarrow X$ is said to be a generalized $F$-contraction if there exists a $\tau>0$ such that

$$
d\left(f x, f^{2} x\right)>0 \Longrightarrow \tau+F\left(d\left(f x, f^{2} x\right)\right) \leq F(d(x, f x))
$$

for all $x \in X$.

Definition 4. Let $(X, d)$ be a metric space and $F \in F$. A mapping $f: X \rightarrow X$ is said to be $F$-nonexpansive if

$$
d(f x, f y)>0 \Longrightarrow F(d(f x, f y)) \leq F(d(x, y)),
$$

for all $x, y \in X$.

Remark 5. It follows from condition (C1) that if $F \in F$ and $f$ is an $F$-nonexpansive self-mapping of a metric space $(X, d)$, then $f$ is nonexpansive (recall that $f$ is nonexpansive provided that $d(f x, f y) \leq d(x, y)$ for all $x, y \in X)$. Conversely, it is clear, by $(\mathrm{C} 1)$, that if $f$ is a nonexpansive selfmapping of a metric space $(X, d)$, then $f$ is $F$-nonexpansive for all $F \in F$.

By considering different choices of mappings $F$ in (2), (3), and (4), we obtain a variety of contractions. For details we refer to [21] and the following examples.

Example 6. Let $(X, d)$ be a metric space, $F \in F$, and let $G$ : $\mathbb{R}_{+} \rightarrow \mathbb{R}$ be given by $G(\alpha)=F(\alpha)-\tau$, where $\tau>0$. It is clear that $G \in F$. Now, if $f: X \rightarrow X$ is a generalized $F$-contraction, then it is a generalized $G$-contraction because for any $x, y \in X$ with $d\left(f x, f^{2} x\right)>0$, we have

$$
\begin{aligned}
\tau+G\left(d\left(f x, f^{2} x\right)\right) & =F\left(d\left(f x, f^{2} x\right)\right) \\
& \leq F(d(x, f x))-\tau=G(d(x, f x)) .
\end{aligned}
$$

Similarly, if $f$ is an $F$-contraction, then it is a $G$-contraction. Furthermore, if $f$ is $F$-nonexpansive then

$$
\begin{aligned}
G(d(f x, f y)) & =F(d(f x, f y))-\tau \\
& \leq F(d(x, y))-2 \tau \leq G(d(x, y))-\tau \\
& \leq G(d(x, y)),
\end{aligned}
$$

whenever $d(f x, f y)>0$, which shows that $f$ is $G^{-}$ nonexpansive. Finally, note that taking $G(\alpha)=\ln (\alpha)$ in (6), we deduce that $f$ is nonexpansive.

Example 7. Let $(X, d)$ be a metric space, let $F_{1}: \mathbb{R}_{+} \rightarrow \mathbb{R}$ be given by $F_{1}(\alpha)=\ln (\alpha)$, and let $f: X \rightarrow X$ be a generalized $F$-contraction. Since $F_{1} \in F$, then (3) becomes

$$
\tau+\ln \left(d\left(f x, f^{2} x\right)\right) \leq \ln (d(x, f x))
$$

whenever $d\left(f x, f^{2} x\right)>0$, which implies

$$
\ln \frac{d\left(f x, f^{2} x\right)}{d(x, f x)} \leq-\tau, \quad \text { that is, } \frac{d\left(f x, f^{2} x\right)}{d(x, f x)} \leq e^{-\tau},
$$

and thus $d\left(f x, f^{2} x\right) \leq e^{-\tau} d(x, f x)$. Hence our definition is more general than those given in $[18,20]$.

If we take $F_{2}(\alpha)=\ln (\alpha)+\alpha$, it is clear that $F_{2} \in F$, and then (2) becomes

$$
\begin{array}{r}
\tau+\ln \left(d\left(f x, f^{2} x\right)\right)+d\left(f x, f^{2} x\right) \leq \\
\ln (d(x, f x)) \\
+d(x, f x)
\end{array}
$$

whenever $d\left(f x, f^{2} x\right)>0$, which implies that

$$
\frac{d\left(f x, f^{2} x\right)}{d(x, f x)} e^{d\left(f x, f^{2} x\right)-d(x, f x)} \leq e^{-\tau}
$$

that is,

$$
d\left(f x, f^{2} x\right) \leq \frac{e^{-\tau}}{e^{d\left(f x, f^{2} x\right)-d(x, f x)}} d(x, f x) .
$$

Definition 8. Let $C$ be a closed subset of metric space $(X, d)$. Then $f: C \rightarrow C$ is called compact if for every bounded subset $A$ of $C, \overline{f(A)}$ is compact in $C$.

Definition 9. If $f: X \rightarrow X$ is a mapping with $f(C) \subseteq C$, then $C$ is called an $f$-invariant subset of $X$.

Definition 10. Let $C$ be a subset of metric space $(X, d)$. As usual, for any $x \in X$, we define

$$
\begin{gathered}
d(x, C)=\inf \{d(x, y): y \in C\}, \\
P_{C}(x)=\{y \in C: d(x, y)=d(x, C)\} .
\end{gathered}
$$

$P_{C}(x)$ is called the set of best approximations of $x$ from $C$. If for each $x \in X, P_{C}(x)$ is nonempty, then $C$ is called proximal. Observe that if $C$ is closed, then $P_{C}(x)$ is also closed.

Definition 11. Let $E$ be a linear space over $\mathbb{R}$. A subset $C$ of $E$ is called star-shaped if there exists at least one point $z \in C$ such that $t z+(1-t) x \in C$ for all $x \in C$ and $0<t<1$. In this case $z$ is called a star centre of $C$.

Let $(X, d)$ be a metric space, $C$ a closed subset of $X$, and $f: C \rightarrow C$ a self-mapping. For each $x \in C$, the set 
$O(x)=\left\{x, f x, \ldots, f^{n} x, \ldots\right\}$ is called the orbit of $x$ (compare [23]). The mapping $f$ is called orbitally continuous at $p$ if $\lim _{n \rightarrow \infty} f^{n} x=p$ implies $\lim _{n \rightarrow \infty} f^{n+1} x=f p$, and $f$ is orbitally continuous on a set $C$ if $f$ is orbitally continuous for all $p \in C$.

\section{Main Results}

In the following a normed linear space $(E,\|\cdot\|)$ will be simply denoted by $E$ if no confusion arises. Furthermore, by a complete subset of a normed linear space $E$ we will mean a subset $A$ of $E$ such that the restriction to $A$ of the metric induced on $E$ by its norm is complete. Of course, every complete subset of a normed linear space is closed, and every closed subset of a Banach space is complete.

Our main result (Theorem 13 below) will be proved with the help of the following re-formulation of Theorem 4 of [22].

Theorem 12 (see [22]). Let $(X, d)$ be a complete metric space, $F \in F$, and $f: X \rightarrow X$ an orbitally continuous generalized F-contraction. Then $f$ has a fixed point.

Theorem 13. Let $E$ be a normed linear space, $C$ a complete and star-shaped subset of $E$, and $F \in F$. If $f: C \rightarrow C$ is an $F$-nonexpansive mapping and $\overline{f(C)}$ is compact, then $f$ has a fixed point.

Proof. We first note that, by Remark 5, $f$ is nonexpansive on $C$, so it is continuous on $C$.

Now let $z$ be a star centre of $C$. For each $n \geq 1$, define $f_{n}: C \rightarrow C$ by

$$
f_{n} x=\left(1-k_{n}\right) z+k_{n} f x
$$

for all $x \in C$, where $0<k_{n}<1$ and $\lim _{n \rightarrow \infty} k_{n}=1$. From the fact that $f$ is continuous on $C$ it immediately follows that each $f_{n}$ is continuous on $C$.

For any fixed $n \geq 1$ and any $x \in C$, we have

$$
\begin{gathered}
F\left(\left\|f_{n} x-f_{n}^{2} x\right\|\right)=F\left(\|\left(1-k_{n}\right) z+k_{n} f x\right. \\
\left.\quad-f_{n}\left(\left(1-k_{n}\right) z+k_{n} f x\right) \|\right) \\
=F\left(\|\left(1-k_{n}\right) z+k_{n} f x-\left(1-k_{n}\right) z\right. \\
\left.\quad-k_{n} f\left(\left(1-k_{n}\right) z+k_{n} f x\right) \|\right) \\
=F\left(\left\|k_{n}\left(f x-f\left(\left(1-k_{n}\right) z+k_{n} f x\right)\right)\right\|\right) .
\end{gathered}
$$

Since $F$ is strictly increasing, with $k_{n}<1$ for each $n \geq 1$, and $f$ is $F$-nonexpansive, we have

$$
\begin{aligned}
F\left(\left\|f_{n} x-f_{n}^{2} x\right\|\right) & <F\left(\left\|f x-f\left(\left(1-k_{n}\right) z+k_{n} f x\right)\right\|\right) \\
& <F\left(\left\|x-\left(\left(1-k_{n}\right) z+k_{n} f x\right)\right\|\right) \\
& =F\left(\left\|x-f_{n} x\right\|\right)
\end{aligned}
$$

Hence

$$
F\left(\left\|x-f_{n} x\right\|\right)-F\left(\left\|f_{n} x-f_{n}^{2} x\right\|\right)>0 .
$$

This implies that there exists $\tau_{n}>0$, such that

$$
0<\tau_{n} \leq F\left(\left\|x-f_{n} x\right\|\right)-F\left(\left\|f_{n} x-f_{n}^{2} x\right\|\right) .
$$

Therefore,

$$
\tau_{n}+F\left(\left\|f_{n} x-f_{n}^{2} x\right\|\right) \leq F\left(\left\|x-f_{n} x\right\|\right) .
$$

Hence, $f_{n}$ is a generalized $F$-contraction for each $n \geq 1$. By Theorem 12, for each $n \geq 1$ there exists $x_{n} \in C$ such that $f_{n} x_{n}=x_{n}$. Since $\overline{f(C)}$ is compact, there exist a subsequence $\left\{f x_{n_{i}}\right\}_{i \geq 1}$ of the sequence $\left\{f x_{n}\right\}_{n \geq 1}$, and an $x \in \overline{f(C)}$ such that

$$
x=\lim _{i \rightarrow \infty} f x_{n_{i}} .
$$

In fact $x \in C$ because $f(C) \subseteq C$ and $C$ is closed.

Since $\lim _{i \rightarrow \infty} k_{n_{i}}=1$ and $x_{n_{i}}=f_{n_{i}} x_{n_{i}}$ for all $i \geq 1$, we deduce that

$$
\begin{aligned}
x & =\lim _{i \rightarrow \infty} f x_{n_{i}}=\lim _{i \rightarrow \infty}\left(\left(1-k_{n_{i}}\right) z+k_{n_{i}} f x_{n_{i}}\right) \\
& =\lim _{i \rightarrow \infty} f_{n_{i}} x_{n_{i}}=\lim _{i \rightarrow \infty} x_{n_{i} .}
\end{aligned}
$$

Therefore,

$$
f x=\lim _{i \rightarrow \infty} f x_{n_{i}}
$$

We conclude that $x=f x$.

Remark 14. Note that, by Remark 5, we can restate the preceding theorem as follows. Let $E$ be a normed linear space (Banach space, resp.) and $C$ a complete (closed, resp.) and star-shaped subset of $E$. If $f: C \rightarrow C$ is a nonexpansive mapping and $\overline{f(C)}$ is compact, then $f$ has a fixed point.

The following is an example where we can apply Theorem 13 to every $F \in F$.

Example 15. Let $\ell_{1}$ be the linear space of all summable sequences of real numbers. Then, the pair $\left(\ell_{1},\|\cdot\|_{1}\right)$ is a (classical) Banach space, where $\|\cdot\|_{1}$ is the norm on $\ell_{1}$ such that, for each $\mathbf{x}:=\left\{x_{n}\right\}_{n \geq 1} \in \ell_{1}$,

$$
\|\mathbf{x}\|_{1}=\sum_{n=1}^{\infty}\left|x_{n}\right| .
$$

In the following any element $\mathbf{x}:=\left\{x_{n}\right\}_{n \geq 1}$ of $\ell_{1}$ will be also denoted as $\left(x_{1}, x_{2}, x_{3}, \ldots, x_{n}, \ldots\right)$.

Let $C$ be the closed unit ball of $\left(\ell_{1},\|\cdot\|_{1}\right)$; that is,

$$
C=\left\{\mathbf{x} \in \ell_{1}:\|\mathbf{x}\|_{1} \leq 1\right\} \text {. }
$$

It is well known that $C$ is a (noncompact) closed subset of $\left(\ell_{1},\|\cdot\|_{1}\right)$. Moreover $C$ is star-shaped with $\mathbf{z}=\mathbf{0}$ a star center of $C$.

Now let $k \in(0,1]$ be constant, and define $f: C \rightarrow C$ as

$$
f \mathbf{x}=\left(x_{1}, k x_{2}, 0, \ldots, 0, \ldots\right),
$$


for all $\mathbf{x}:=\left(x_{n}\right)_{n \geq 1} \in \ell_{1}$. Clearly $f$ is nonexpansive on $C$, and hence it is $F$-nonexpansive for any $F \in F$, by Remark 5 .

Furthermore, $f(C)$ is homeomorphic to the compact subset of $\mathbb{R}^{2}$,

$$
\left\{(u, v) \in \mathbb{R}^{2}:|u|+\left|\frac{v}{k}\right| \leq 1\right\}
$$

so that $f(C)$ is compact. Hence $f(C)=\overline{f(C)}$ and thus $\overline{f(C)}$ is compact. We have shown that all conditions of Theorem 13 (compare Remark 14) are satisfied. Thus $f$ has a fixed point. In fact, the fixed points of $f$ are the elements $\mathbf{x}=\left\{x_{n}\right\}_{n \geq 1}$ of $C$ such that $x_{n}=0$ whenever $n \geq 2$.

Now we give an example of a discontinuous self-mapping $f$ on a compact metric space which is a generalized $F$ contraction but not an $F$-contraction. So the class of generalized $F$-contraction mappings is bigger than the class of F-contraction.

Example 16. Let $X=\mathbb{R}$, and let $C=[0,1]$ be endowed with usual metric. Define a mapping $f: C \rightarrow C$ as $f(x)=1$ if $x \in\{0,1\}$, and $f(x)=x / 2$ if $x \in(0,1)$.

Let $F: \mathbb{R}_{+} \rightarrow \mathbb{R}$ be defined by $F x=\ln x$ for $x \in(0,1)$. Note that

$$
\ln 2+F\left(d\left(f x, f^{2} x\right)\right) \leq F(d(x, f x))
$$

is satisfied for all $x \in C$ whenever $d\left(f x, f^{2} x\right)>0$. Hence, $f$ is a generalized $F$-contraction. Clearly $f$ is not continuous at $x=1$ and at $x=0$. Hence, $f$ is not an $F$-contraction (see Remark 2.1 in [21] ) or let $x=0$ and $y=1 / 2$; then

$$
\begin{aligned}
F\left(d\left(f 0, f \frac{1}{2}\right)\right) & =F\left(d\left(1, \frac{1}{4}\right)\right) \\
& =F\left(\frac{3}{4}\right)=\ln \left(\frac{3}{4}\right)=\ln 3-2 \ln 2,
\end{aligned}
$$

while

$$
F\left(d\left(0, \frac{1}{2}\right)\right)=F\left(\frac{1}{2}\right)=\ln \left(\frac{1}{2}\right)=-\ln 2,
$$

and thus

$$
F\left(d\left(0, \frac{1}{2}\right)\right)-F\left(d\left(f 0, f \frac{1}{2}\right)\right)=\ln 2-\ln 3<0 .
$$

Hence, there does not exist any $\tau>0$, such that, for $x=0$ and $y=1 / 2$,

$$
\tau+F(d(f x, f y)) \leq F(d(x, y))
$$

is satisfied. Now let $x_{0}=0$; then $f(0)=1$, and $f^{2}(0)=1$. Hence, the orbit of $x_{0}$ is the set $O(0)=\{0,1,1, \ldots\}=\overline{O(0)}$ which is compact and

$$
\lim _{n \rightarrow \infty} f^{n}(0)=1, \quad \lim _{n \rightarrow \infty} f^{n+1}(0)=f 1=1,
$$

that is $f$ is orbitally continuous at 1 . Hence, by Theorem $12, f$ has a fixed point (in fact $x=1$ is the only fixed point of $f$ ).
In the last part of the paper we discuss nonemptiness and existence of fixed points for the set of best approximations of closed subsets of metric spaces and of normed spaces, respectively.

Theorem 17. Let $(X, d)$ be a metric space. Let $F \in F$ be a continuous mapping and let $f: X \rightarrow X$ be F-nonexpansive with a fixed point $u \in X$. If $C$ is a closed $f$-invariant subset of $X$ such that $f$ is compact on $C$, then the set $P_{C}(u)$ of best approximations is nonempty.

Proof. Let $r=d(u, C)$. Then, there is a sequence $\left\{y_{n}\right\}_{n \geq 1}$ in $C$ such that $\lim _{n \rightarrow \infty} d\left(u, y_{n}\right)=r$. Since $\left\{y_{n}: n \geq 1\right\}$ is a bounded subset of $C$ and $f$ is compact on $C$, the set $\overline{\left\{f y_{n}: n \geq 1\right\}}$ is a compact subset of $C$, and so there exist a subsequence $\left\{f y_{n_{i}}\right\}_{i \geq 1}$ of $\left\{f y_{n}\right\}_{n \geq 1}$ and an $x \in C$ such that $\lim _{i \rightarrow \infty} f y_{n_{i}}=x$. Now,

$$
\begin{aligned}
F(r) \leq F(d(u, x)) & =F\left(\lim _{i \rightarrow \infty} d\left(f u, f y_{n_{i}}\right)\right) \\
& =\lim _{i \rightarrow \infty} F\left(d\left(f u, f y_{n_{i}}\right)\right) \\
& \leq \lim _{i \rightarrow \infty} F\left(d\left(u, y_{n_{i}}\right)\right) \\
& =F\left(\lim _{i \rightarrow \infty} d\left(u, y_{n_{i}}\right)\right)=F(r) .
\end{aligned}
$$

This implies

$$
F(r)=F(d(u, x))
$$

Since $F$ is strictly increasing, we get $r=d(u, x)$. Hence $P_{C}(u)$ is nonempty.

As an application of Theorems 13 and 17, we deduce the following.

Theorem 18. Let $E$ be a normed linear space. Let $F \in F$ be a continuous mapping and let $f: E \rightarrow E$ be F-nonexpansive with a fixed point $u \in E$. If $C$ is a complete $f$-invariant subset of $E$ such that $f$ is compact on $C$, and $P_{C}(u)$ is a star-shaped set, then $f$ has a fixed point in $P_{C}(u)$.

Proof. By Theorem 17, $P_{C}(u)$ is nonempty. We show that $P_{C}(u)$ is $f$-invariant. To this end, let $y \in P_{C}(u)$ and set $r=d(u, C)$; then

$$
\begin{aligned}
F(r) & \leq F(d(u, f y))=F(d(f u, f y)) \\
& \leq F(d(u, y))=F(r) .
\end{aligned}
$$

This implies

$$
F(r)=F(d(u, f y)) .
$$

Since $F$ is strictly increasing, we get $r=d(u, f y)$. So $f y \in$ $P_{C}(u)$. This proves that $P_{C}(u)$ is $f$-invariant, so $f: P_{C}(u) \rightarrow$ $P_{C}(u)$ is $F$-nonexpansive. Now observe that if $C$ is complete, then $P_{C}(u)$ is also complete. Hence, $P_{C}(u)$ is star-shaped and complete, and $\overline{f\left(P_{C}(u)\right)}$ is compact, so, by Theorem 13 , there exists $x \in P_{C}(u)$ such that $f x=x$. 
Remark 19. As in the case of Theorem 13 (see Remark 14), the preceding theorem can be restated as follows. Let $E$ be a normed linear space (Banach space, resp.) and let $f: E \rightarrow E$ be nonexpansive with a fixed point $u \in E$. If $C$ is a complete (closed, resp.) $f$-invariant subset of $E$ such that $f$ is compact on $C$ and $P_{C}(u)$ is a star-shaped set, then $f$ has a fixed point in $P_{C}(u)$.

We conclude the paper illustrating Theorem 18 with the following example.

Example 20. Let $\left(\ell_{1},\|\cdot\|_{1}\right)$ be the Banach space of Example 15. Define $f: \ell_{1} \rightarrow \ell_{1}$ as

$$
f \mathbf{x}=\left(x_{1}, k x_{2}, 0, \ldots, 0, \ldots\right),
$$

for all $\mathbf{x}:=\left\{x_{n}\right\}_{n \geq 1} \in \ell_{1}$, with $k \in(0,1]$. Let $F \in$ $F$ be continuous. Since $f$ is nonexpansive, it follows from Remark 5 that it is $F$-nonexpansive. Of course, $f$ has fixed points. In fact

$$
\operatorname{Fix}(f)=\left\{\mathbf{x}:=\left\{x_{n}\right\}_{n \geq 1} \in \ell_{1}: x_{n}=0 \forall n \geq 2\right\} .
$$

As in Example 15, let $C$ be the closed unit ball of $\left(\ell_{1},\|\cdot\|_{1}\right)$. We know that $C$ is a closed and thus complete, $f$-invariant subset of $E$ such that $f$ is compact on $C$.

Then, if we choose $\mathbf{x}:=\left\{x_{n}\right\}_{n \geq 1} \in \operatorname{Fix}(f)$ such that $\left|x_{1}\right|>1$, we deduce that $P_{C}(\mathbf{x})=(1,0,0, \ldots, 0, \ldots)$ if $x_{1}>1$, and $P_{C}(\mathbf{x})=(-1,0,0, \ldots, 0, \ldots)$ if $x_{1}<-1$. Therefore, $P_{C}(u)$ is trivially star-shaped. Thus, all conditions of Theorem 18 (compare Remark 19) have been verified.

\section{Conflict of Interests}

The authors declare that there is no conflict of interests regarding the publication of this paper.

\section{Acknowledgments}

The authors are very grateful to the referees for their valuable comments and suggestions, and, in particular, to one of them for calling our attention on the crucial fact stated in the first part of Remark 5 and for the elegant reformulation of Theorem 13 stated in Remark 14. Salvador Romaguera acknowledges the support of the Universitat Politècnica de València, Grant PAID-06-12-SP20120471.

\section{References}

[1] S. Banach, "Sur les opérations dans les ensembles abstraits et leur application aux équations intégrales," Fundamenta Mathematicae, vol. 3, pp. 133-181, 1922.

[2] I. Arandjelović, Z. Kadelburg, and S. Radenović, "Boyd-Wongtype common fixed point results in cone metric spaces," Applied Mathematics and Computation, vol. 217, no. 17, pp. 7167-7171, 2011.

[3] D. W. Boyd and J. S. W. Wong, "On nonlinear contractions," Proceedings of the American Mathematical Society, vol. 20, pp. 458-464, 1969.
[4] L.-G. Huang and X. Zhang, "Cone metric spaces and fixed point theorems of contractive mappings," Journal of Mathematical Analysis and Applications, vol. 332, no. 2, pp. 1468-1476, 2007.

[5] E. Rakotch, "A note on contractive mappings," Proceedings of the American Mathematical Society, vol. 13, pp. 459-465, 1962.

[6] E. Tarafdar, "An approach to fixed-point theorems on uniform spaces," Transactions of the American Mathematical Society, vol. 191, pp. 209-225, 1974.

[7] J. G. Dix and G. L. Karakostas, "A fixed-point theorem for S-type operators on Banach spaces and its applications to boundary-value problems," Nonlinear Analysis: Theory, Methods \& Applications, vol. 71, no. 9, pp. 3872-3880, 2009.

[8] K. Latrach, M. A. Taoudi, and A. Zeghal, "Some fixed point theorems of the Schauder and the Krasnosel'skii type and application to nonlinear transport equations," Journal of Differential Equations, vol. 221, no. 1, pp. 256-271, 2006.

[9] C. Rousseau, "Point fixe de Banach," Accromath 5, hiverprintemps, 2010, (in French), http://www.accromath.ca/.

[10] G. Meinardus, "Invarianz bei linearen Approximationen," Archive for Rational Mechanics and Analysis, vol. 14, pp. 301-303, 1963.

[11] B. Brosowski, "Fixpunktsätze in der Approximationstheorie," Mathematica, vol. 11, no. 34, pp. 195-220, 1969.

[12] L. Habiniak, "Fixed point theorems and invariant approximations," Journal of Approximation Theory, vol. 56, no. 3, pp. 241244, 1989.

[13] T. L. Hicks and M. D. Humphries, "A note on fixed-point theorems," Journal of Approximation Theory, vol. 34, no. 3, pp. 221-225, 1982.

[14] N. Hussain, D. O’Regan, and R. P. Agarwal, "Common fixed point and invariant approximation results on non-star-shaped domain," Georgian Mathematical Journal, vol. 12, no. 4, pp. 659669, 2005.

[15] T. D. Narang, "Applications of fixed point theorems to approximation theory," Matematički Vesnik, vol. 36, no. 1, pp. 69-75, 1984.

[16] S. P. Singh, "An application of a fixed-point theorem to approximation theory," Journal of Approximation Theory, vol. 25, no. 1, pp. 89-90, 1979.

[17] A. Smoluk, "Invariant approximations," Matematyka Stosowana, vol. 17, pp. 17-22, 1981.

[18] P. V. Subrahmanyam, "An application of a fixed point theorem to best approximation," Journal of Approximation Theory, vol. 20, no. 2, pp. 165-172, 1977.

[19] W. G. Dotson," "Fixed point theorems for non-expansive mappings on star-shaped subsets of Banach spaces," Journal of the London Mathematical Society, vol. 4, pp. 408-410, 1972.

[20] L. A. Khan and A. R. Khan, "An extension of BrosowskiMeinardus theorem on invariant approximation," Approximation Theory and its Applications, vol. 11, no. 4, pp. 1-5, 1995.

[21] D. Wardowski, "Fixed points of a new type of contractive mappings in complete metric spaces," Fixed Point Theory and Applications, vol. 2012, article 94, 6 pages, 2012.

[22] M. Abbas, B. Ali, and S. Romaguera, "Fixed and periodic points of generalized contractions in metric spaces," Fixed Point Theory and Applications, vol. 2013, article 243, 11 pages, 2013.

[23] T. L. Hicks and B. E. Rhoades, "A Banach type fixed-point theorem," Mathematica Japonica, vol. 24, no. 3, pp. 327-330, 1979. 


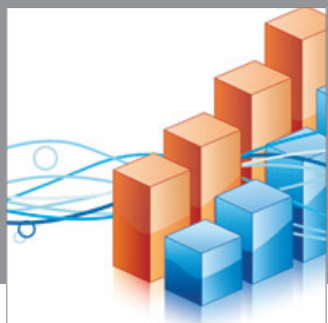

Advances in

Operations Research

mansans

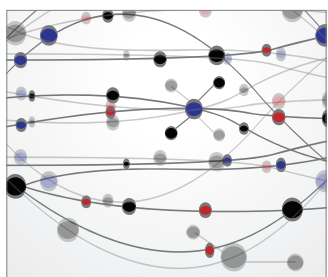

The Scientific World Journal
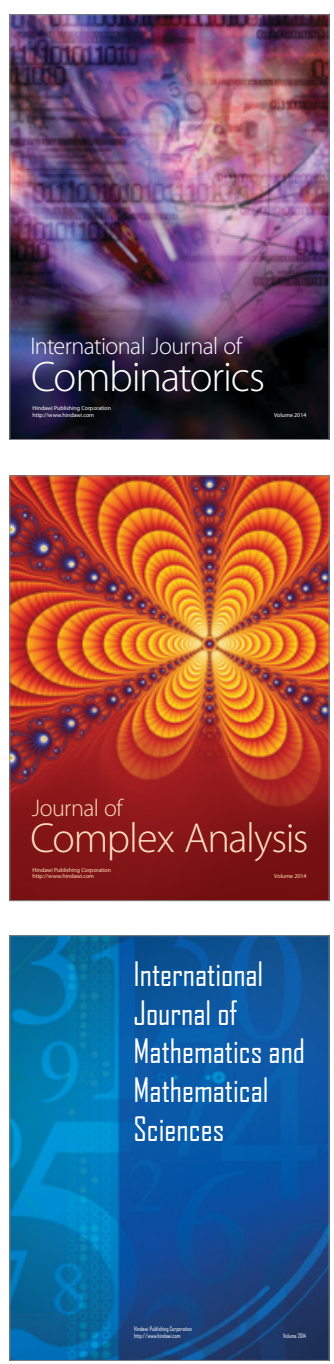
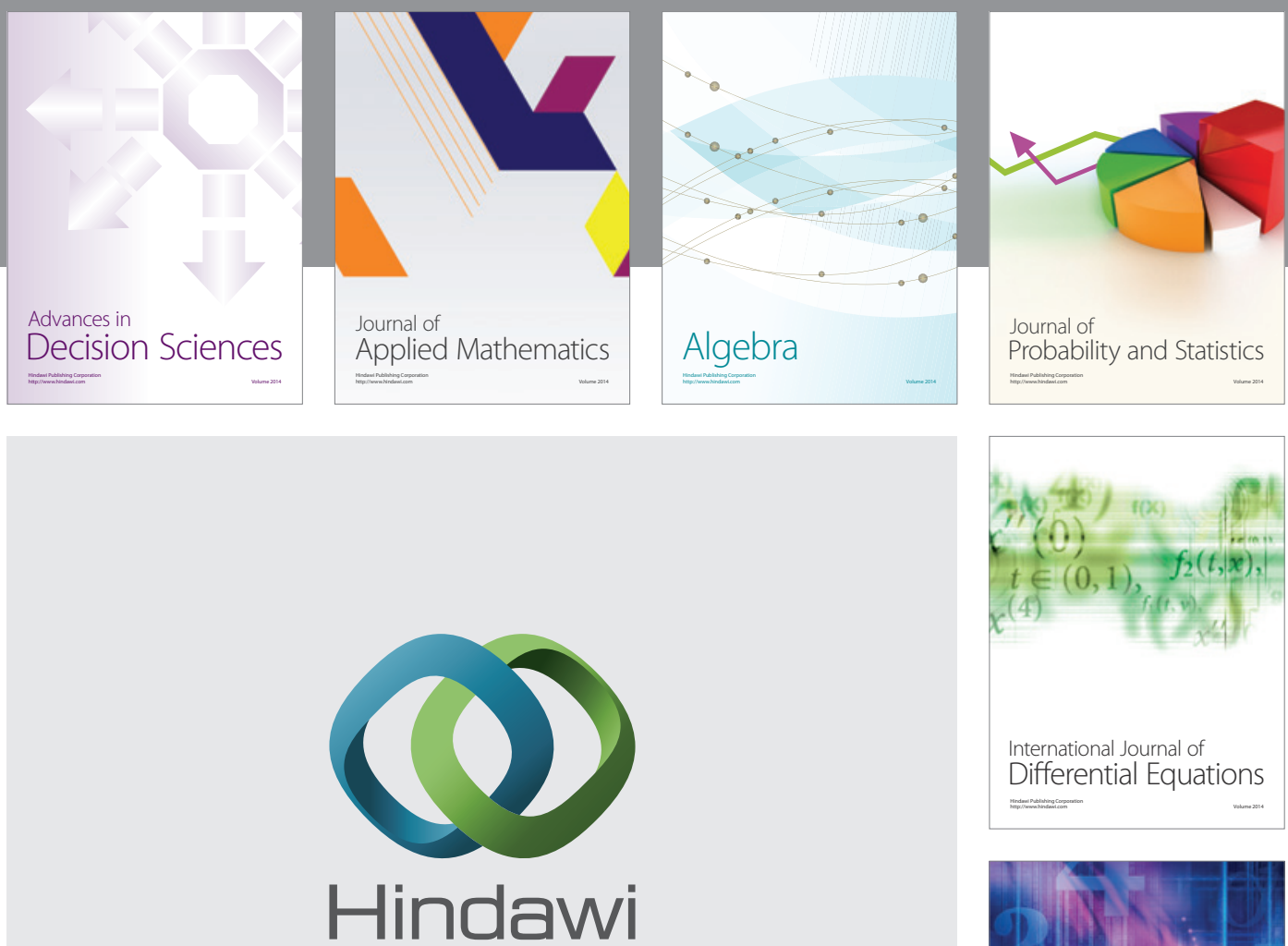

Submit your manuscripts at http://www.hindawi.com
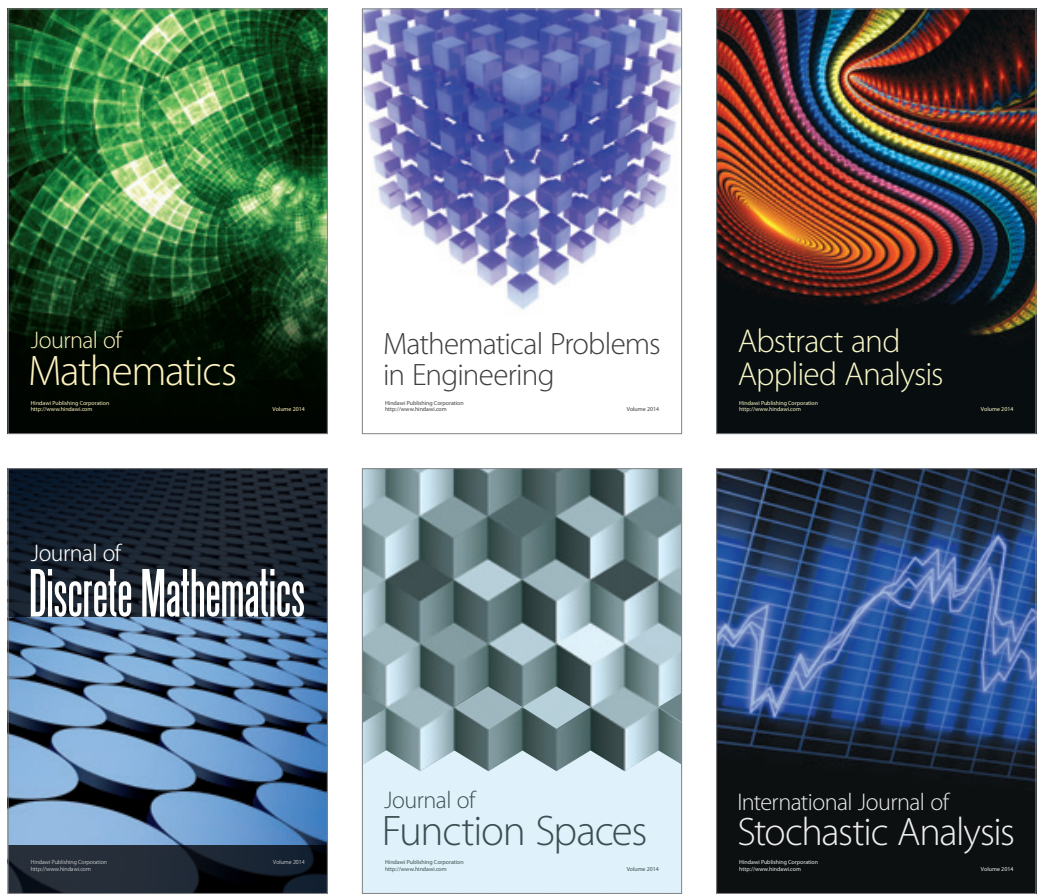

Journal of

Function Spaces

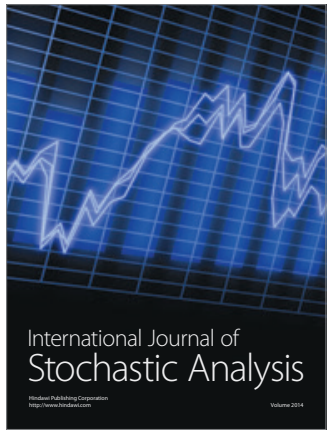

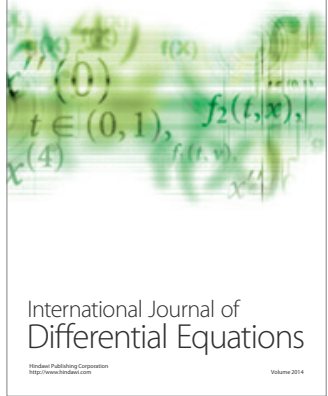
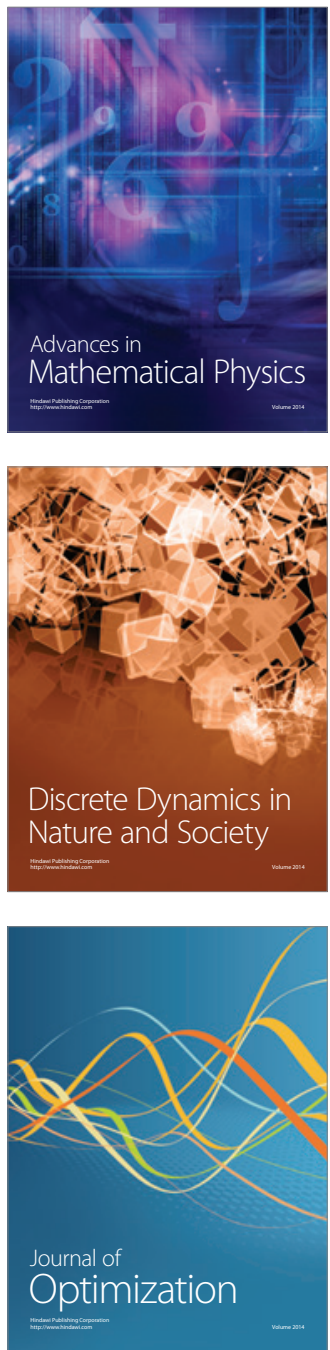